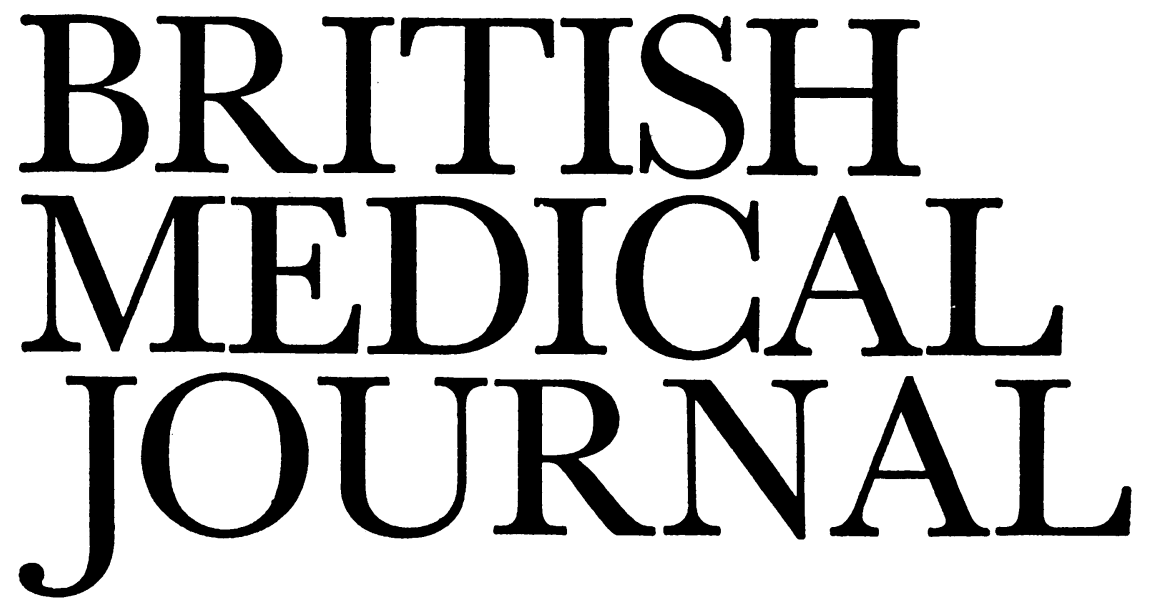

\title{
Significance of Ectopic Beats
}

More than 20 years ago the late Paul Wood stated, "On the whole . . . it is wise to assume the innocence of ectopic beats under any conditions, and to judge organic disease on other grounds."1 Ectopic beats are common. They often arise from an irritable ventricular, sometimes supraventricular, focus and cause premature depolarization of the ventricles. Usually the premature beat leaves the ventricles refractory to further excitation for long enough to miss the next arrival of the normally conducted atrial excitation, so the average number of beats per minute does not change. At the wrist a beat may be missing if the aortic valve either fails to open or opens only slightly. True extra systoles are less common. The frequent occurrence of ectopic beats in hearts which cannot be shown by any other criterion to be diseased means that clinicians are generally trained to attach little significance in ambulant patients to occasional ectopic beats of any sort.

Doubtless this is a healthy attitude, yet ventricular ectopic beats are seen in more than half of patients in the acute phase of a myocardial infarct and are then presumably symptomatic of myocardial disease. Much of the worldwide investment in monitoring equipment for coronary care units is based on two assumptions: (1) that ventricular fibrillation may be preceded by increased numbers of ventricular ectopic beats, which anyone can be quickly trained to recognize on an oscilloscope screen; and (2) that timely treatment-for example, with lignocaine may stave off ventricular fibrillation. Though there is at present some doubt about these assumptions, it is certainly true that ectopic beats are much commoner after an acute myocardial injury than at other times. In principle, therefore, it is tempting to suppose that ectopic beats must have some prognostic significance if only we knew accurately how to assess it.

Prospective studies are needed, but only a few have so far been done. A study of a sample population of Tecumseh, Michigan, ${ }^{2}$ showed that 91 out of 97 people who died/ of coronary artery disease during a six-year follow-up period had rhythm or conduction defects on an electrocardiogram previously recorded at rest. However, the difficulty in using this type of information is the very high frequency of ectopic beats and other minor abnormalities among people who do not subsequently have coronary disease. Since exercise increases the heart's requirements for blood and also speeds up its rate, it is logical to expect that minor degrees of ischaemia might show themselves only during exercise in the guise of a disorder of conduction or rhythm, just as more serious ischaemia shows itself by the clinical symptom of precordial pain on exercise. Thus a distinction might be possible between prognostically serious disorders of rhythm and innocent ones by observing the response to exercise, especially since ectopic ventricular beats, which by all other criteria are innocent, often disappear during exercise as the heart accelerates. ${ }^{3}$

J. A. Vedin and his colleagues in Sweden ${ }^{4}$ have recently attempted to correlate the occurrence of ectopic beats at rest and during exercise with the subsequent prognosis in a long-term prospective study. A random and apparently representative sample of about 800 men, all aged 51 in 1963, was re-examined in 1967. Follow-up was remarkably good, and the population sample was re-examined in 1971, at which time nearly $95 \%$ of the men in the sample were still under observation. Only resting electrocardiograms were taken in 1963, but in 1967 the men were given a graded exercise test proceeding to the highest levels of exercise tolerated. Of the total sample $2.6 \%$ had ventricular and $1.9 \%$ had supraventricular ectopic beats at rest, and a few others had other abnormalities of rhythm or conductionfor example, left bundle branch block and atrial fibrillation. These figures compare closely with other published statistics. During exercise some of the normal men in this study developed ectopic beats, and in the whole group some kind of rhythm or conduction defect developed in $11.4 \%$ when maximally stressed by exercise. It is interesting to note that supraventricular ectopic beats were not produced by exercise in any of the men, though they increased in frequency after exercise ceased.

Some indication that exercise-provoked ectopic beats were of pathological significance is provided by their high correlation with ischaemic electrocardiographic abnormalities (for example, depression of the ST segment) developing during exercise, and by their correlation with high blood pressure and high blood-glucose concentrations. But the general contention that ectopic beats are in themselves of little significance was evident from the failure of other symptoms of myocardial ischaemia, such as dyspnoea on effort, angina of effort, and a previous history of myocardial infarction, to correlate with the appearance of ectopic beats 
on exercise. The conclusion is hard to resist that in most cases ectopic beats, even when appearing during exercise, reflect the dysfunction of a usually very small part of the whole myocardium rather than of a great part of it. If this is so, then the correlation one might expect would be perhaps not so much with clinical angina of effort or myocardial infarction but rather with sudden death due to acute ventricular fibrillation, which can apparently occur sometimes without a demonstrable myocardial infarct. 56 That this possibility exists is perhaps shown by the observations of Vedin and colleagues that two of the three men in the cohort who died during 1968-70 had more than 1 in 4 ectopic beats during strenuous exercise, and in both cases the irregularity was accentuated during strenuous exercise. Similarly suggestive observations have previously been recorded. ${ }^{2}$

None of this evidence carries enough statistical weight for firm conclusions. Indeed, most of it reinforces Paul Wood's opinion. It would probably be wise to advise someone having exceedingly frequent ectopic beats made more frequent with exercise to avoid strenuous exertion. But there is not enough evidence to go further than this, and certainly not enough to embark on the wholesale prophylactic prescription of antiarrhythmic drugs for ectopic beats. These still remain, as they have always done, a most unreliable measure of coronary or any other heart disease.

1 Wood, P., Diseases of the Heart and Circulation. London, Eyre and Spottiswoode, 1950

2 Chiang, B. N., Perlman, L. V., Ostrander, L. D., and Epstein, F. H., Annals of Internal Medicine, 1969, 70, 1159

8 Bellet, S., Clinical Disorders of the Heart Beat, 3rd edn., p. 691. Philadelphia, Lea and Febiger, 1971.

4 Vedin, J. A., Wilhelmsoon, C. E., Wilhelmsen, L., Bjure, J., and EkströmJodal, B., American Fournal of Cardiology, 1972, 30, 25.

5 Cantwell, J. D., and Fletcher, G. F., fournal of the American Medical Association, 1969, 210, 130.

- Pyfer, H. R., and Doane, B. L., Fournal of the American Medical Association, 1969, 210, 101 .

\section{Scoliosis}

With the disappearance of tuberculosis and poliomyelitis the frequency and importance of scoliosis has become more apparent. It is very common. In a survey in Edinburgh of more than 11,000 children $R$. Wynne-Davies ${ }^{1}$ found that 3.9 per 1,000 girls had idiopathic scoliosis. Though idiopathic scoliosis is the commonest and most important of the structural scolioses spinal curvature may be seen as a symptom of more than 50 diseases, many of them rare inherited syndromes. Congenital scoliosis due to vertebral anomalies and scoliosis secondary to neurological disease, of which neurofibromatosis is the commonest, are the next most frequent and important causes.

From all these causes therefore scoliosis is common. It is obviously deforming and it is also disabling. Compression of the lungs in scoliosis causes difficulty in aerating the alveoli and circulating blood through the lungs. ${ }^{2} 3$ In consequence cor pulmonale may seriously shorten the lives of those with thoracic curves over $75^{\circ}$.

The rapidity of development and severity of the deformity in idiopathic scoliosis have always been puzzling, because so far as could be discerned the children are otherwise absolutely normal. What is it that can turn a healthy, vigorous young girl of some 10 years of age into a crippled, deformed child with a scoliosis of more than $100^{\circ}$ in less than five years? We do not know.

Despite much study, idiopathic scoliosis remains mysterious, though at last the first glimmers of light and understanding are emerging. An initial study by Miss Wynne-Davies ${ }^{1}$ showed that if the first, second, and third degree relatives of children with idiopathic scoliosis were examined spinal curvature would be found to be 20 times as common among them as in a comparable group of the general population. These figures are about the same as the findings in families of children with congenital club-foot or congenital dislocation of the hip. A more recent study (to be published) showed that in first degree relatives scoliosis was 60 times as common as in the population. This was based on a clinical examination. When the parents were all examined radiographically as well, as was done in 68 families, the incidence of measured curves over $10^{\circ}$ was $11.9 \%$ in the fathers and $23.9 \%$ in the mothers. There can now be no doubt that there is a considerable familial basis to idiopathic scoliosis. Where precisely it acts remains unknown. A curious finding is the frequent association of mental deficiency-16.7\% in the males with scoliosis, excluding infants.

The treatment of scoliosis remains one of the most difficult problems in orthopaedic surgery. In the last decade it may be claimed that in the hands of experts the results have become worthwhile. In all but the benign and minor curves requiring nothing but continuing observation treatment must be by initial correction, followed by spinal fusion. In those too young for fusion the Milwaukee brace has a valuable role in preventing deterioration, and it has a similar but useful role in curves appearing near the end of skeletal maturity, when surgical fusion may thereby prove unnecessary.

The problem is delay. Physiotherapy has long been proved valueless but its use is still delaying effective treatment. The prognosis depends on the aetiology, and the patterns of curvature in relation to each cause are the main guides to the outcome. The course of scoliosis, even of the smallest curve, can be reasonably predicted. ${ }^{4-7}$ Scoliosis of more than $50^{\circ}$ nowadays is almost always due to a delaying error, a failure of recognition. Most scolioses coming to correction and fusion are well over this figure, largely uncorrectable, and shortening life by their effect on the lungs. Additionally by their deforming effect in girls, the main sufferers, they frustrate the expectation of marriage and children for the majority.

For early curves, or curves kept small by timely recognition and the use of the Milwaukee brace, correction with a simple plaster cast followed by fusion is safe and satisfactory. This treatment has few disadvantages and now requires only a few weeks in hospital. Some surgeons prefer as a routine to use the rods devised by P. R. Harrington, 8 though they are not then strictly necessary.

The newer methods of correction, of which Harrington was the first to devise an effective one, are valuable in severe cases or the many difficult and special problems. The halo-pelvic apparatus ${ }^{9}$ and the Dwyer cable and screw ${ }^{10}$ used after vertebral osteotomy have become popular. A disturbing number of paraplegias is being reported after all these powerful methods of treatment and their place has yet to be defined, but used with care they have an important role. Myelography for the detection of diastematomyelia and other anomalies in relation to the cord seems now to be a preoperative necessity.

As in all other orthopaedic deformities, such as polio- 